\title{
Different tumoricidal effects of interferon subclasses and p53 status on hepatocellular carcinoma development and neovascularization
}

\author{
RYUICHI NOGUCHI, HITOSHI YOSHIJI, YASUHIDE IKENAKA, MITSUTERU KITADE, \\ KOSUKE KAJI, JUNICHI YOSHII, KOJI YANASE, TADASHI NAMISAKI, MASAHARU YAMAZAKI, \\ TATSUHIRO TSUJIMOTO, TAKEMI AKAHANE, MASAHITO UEMURA and HIROSHI FUKUI
}

Third Department of Internal Medicine, Nara Medical University, Nara, Japan

Received August 21,2007; Accepted October 3, 2007

\begin{abstract}
Interferon (IFN) is known as a multifunctional cytokine. The aim of this study was to examine the different effects of IFN subclass; namely, IFN- $\alpha$ and IFN- $\beta$, on hepatocellular carcinoma (HCC) growth especially in conjunction with angiogenesis that is known to play a pivotal role in the tumor growth. Furthermore, we also examined whether the p53 status in the tumor would alter the anti-tumoral effect of IFN against HCC growth since the p53 status reportedly affected the therapeutic effect of anti-angiogenic agents against cancer. When compared with IFN- $\alpha$, IFN- $\beta$ exerted a more potent inhibitory effect on HCC growth, even after the tumor was established, along with suppression of neovascularization in the tumor. A single treatment with clinically comparable low doses of IFN- $\beta$ significantly inhibited HCC growth whereas the same dose of IFN- $\alpha$ did not. IFN- $\beta$ also significantly suppressed the tumor growth both in the p53wild and p53-mutant HCC cells. Our in vitro study revealed that IFN- $\beta$ showed a more potent inhibitory effect on the endothelial cell proliferation than IFN- $\alpha$ as in the in vivo study. Collectively, IFN may be an alternative anti-angiogenic agent against HCC since it exerted a significant tumoricidal effect regardless of the host p53 status even at a low dose. A cautious approach may be also required in the clinical practice since even in a same IFN subclass (class-I), IFN- $\alpha$ and IFN- $B$ exert tumoricidal effects of different magnitudes on HCC.
\end{abstract}

\section{Introduction}

Hepatocellular carcinoma (HCC) is now the fifth most common cancer worldwide, and its incidence will further increase

Correspondence to: Dr Hitoshi Yoshiji, Third Department of Internal Medicine, Nara Medical University, Shijo-cho 840, Kashihara, Nara 634-8522, Japan

E-mail: yoshijih@naramed-u.ac.jp

Key words: angiogenesis, interferon, hepatocellular carcinoma, p53, vascular endothelial cell growth factor accounting for 500,000 new cases annually (1). Despite the available therapeutic options, the incidence is still nearly equal to the mortality rate. At present, liver transplantation is considered the only curative option for HCC. However, it is not feasible to apply this option for all patients with HCC since the number of donors is absolutely insufficient. Several other modalities, such as surgery, percutaneous ethanol injection (PEI), transcatheter arterial embolization (TAE), and radiofrequency ablation (RFA), are reportedly useful to improve the prognosis in patients with small HCC (1). For advanced HCC, chemotherapy is the only remaining option although its efficacy is very poor so far (2). Recently, the progress in implantable drug delivery system has allowed repeated arterial infusion of chemotherapeutic agents, such as cisplatin (CDDP) and 5-fluouracil (5-FU), for patients with advanced HCC (3). An alternative agent; namely, interferon (IFN), has also been reported to improve the survival rate in combination with 5-FU (4,5).

IFNs are a family of natural glycoproteins initially discovered on their basis of antiviral activity. These cytokines have various biological properties, including immunomodulation and anti-proliferative actions (6). Type-I IFN, IFN- $\alpha$ and IFN- $\beta$ have been widely used for the treatment of patients with chronic hepatitis $\mathrm{C}$ in the clinical practice $(7,8)$. There is a growing body of evidence that IFN reduces the incidence of $\mathrm{HCC}$ in patients with chronic hepatitis $\mathrm{C}$ and the intrahepatic recurrence of HCC (9). Furthermore, several reports demonstrated a drastic $\mathrm{HCC}$ regression after IFN therapy (10). However, to date, few studies have shown tumoricidal differences between IFN- $\alpha$ and IFN- $\beta$.

Any solid tumor that has not acquired its new own blood supply can not grow to more than only a few millimeters in size, including HCC (11). One of the characteristic features of HCC in the clinical practice is hypervascularity. Several studies have shown that neovascularization and angiogenic factors, such as the vascular endothelial cell growth factor (VEGF), are significantly up-regulated in the human HCC samples $(12,13)$. We previously reported that angiogenesis plays a pivotal role in the murine HCC development, and that suppression of the VEGF-signaling pathway markedly attenuated the tumor growth (14). IFN also has an antiangiogenic activity both in vitro and in vivo (15). Although IFN- $\alpha$ and IFN- $\beta$ reportedly exert different anti-angiogenic 
activities in vitro $(16,17)$, the in vivo anti-angiogenic differences in HCC have not been reported yet.

HCC is molecularly complex, as nearly every carcinogenic mechanism is altered to some degree, and HCC cells harbor numerous genetic defects such as p53 (18). Mutations of p53 gene are detectable in $\sim 50 \%$ of HCC especially in the late stage of HCC (19). Loss of the p53 gene function is associated with poorly differentiated HCC and a shorter survival time (18). It has been reported that the host p53 status affects the tumor response to anti-angiogenic therapy, and that transcription of p53 gene is induced by IFN (20). It is very important to examine whether the anti-angiogenic therapeutic effect of IFN is affected by the host tumoral p53 status or not for future clinical application.

In the present study, to evaluate the feasibility of future clinical application, we examined the different effects of IFN- $\alpha$ and IFN- $\beta$ at clinically comparable low doses on the experimental HCC tumor development and angiogenesis. We also elucidated whether the p53 status in the tumor alters the anti-tumoral effect of IFN against HCC growth, and investigated the possible mechanisms involved.

\section{Materials and methods}

Compounds and cell lines. IFN- $\alpha$ and IFN- $\beta$ were generously supplied by Hayashibara Biochemical Laboratories, Inc. (Okayama, Japan) and Toray Industries, Inc. (Tokyo, Japan), respectively. The p53-wild and -mutant HCC cell lines, BNL.1 ME A.7R.1 (BNL) and PLC/PRF/5 (PLC) were obtained from the Japanese Cancer Research Resources Bank (Tokyo, Japan). The primary human umbilical endothelial cells (EC) were purchased from Kurabo (Osaka, Japan). These cells were cultured in the respective recommended medium as described previously (21).

Animal treatment. A total of 60 male 6-week-old BALB/c $(n=30)$ and BALB/c-nu/nu $(n=30)$ mice were purchased from Japan SLC Inc. (Hamamatsu, Shizuoka, Japan). They were housed under controlled temperature conditions and relative humidity, with 10-15 air changes per hour (h) and light illumination for $12 \mathrm{~h}$ a day. To compare the tumoricidal difference between IFN- $\alpha$ and IFN- $\beta$, we transplanted $5 \times 10^{6}$ PLC cells into the flank of BALB/c-nu/nu mice. The mice were randomly divided into 4 groups ( $\mathrm{n}=10$ in each group). After the mean tumor volume reached $200 \mathrm{~mm}^{3}$, the mice of the phosphate buffer saline (PBS)-treated group served as a control (Cont). The mice in IFN- $\alpha$ group and IFN- $\beta$ group received $1 \times 10^{4} \mathrm{IU}$ of murine IFN- $\alpha$ and $-\beta$, respectively, twice a week with subcutaneous injection on the contralateral side of the tumor. The doses of these agents are reportedly almost comparable to those used in the clinical practice (22).

The next experiment was conducted to examine the effect of IFN- $B$ on BNL tumor growth. In this experiment, administration of IFN- $\beta$ started on day 0 , and IFN treatment was similar to that of the PLC experiment. The tumor was measured twice a week as described previously (22), and the animals were allowed free access to food and water throughout the acclimation and experiment protocols. The mice were sacrificed 56 and 32 days after the tumor cell implantation in the PLC and BNL experiments, respectively. All animal procedures were performed according to approved protocols and in accordance with the recommendations for the proper care and use of laboratory animals.

Neovascularization in the tumor. To evaluate the expression of CD31 mRNA, which is widely used as neovascularization marker, we performed a semi-quantitative RT-PCR analysis. Tumors were immediately snap-frozen for RNA extraction $(\mathrm{n}=5$ in each experimental group), and mRNA was extracted from the pool of tumors. The primer for the mouse CD31 was follows: sense, 5'-CGGTGGATGAAGTTGTGATT-3'; anti-sense, 5'-ACCGTCTCTTGTGGCTCTCGT-3'. PCR was performed at $94^{\circ} \mathrm{C}$ for $1 \mathrm{~min}$, at $52^{\circ} \mathrm{C}$ for $1 \mathrm{~min}$, and at $72^{\circ} \mathrm{C}$ for $1 \mathrm{~min}$ for 30 cycles. To prevent genomic DNA contamination, all RNA samples were subjected to DNase I digestion and checked by 30 cycles of PCR to confirm the absence of any amplified DNA. The PCR products (620 bp) were analyzed by electrophoresis on $1.5 \%$ agarose gel, and the products were visualized by staining with ethidium bromide. Densitometric analysis was performed by measuring the absorbance of each band with Fuji BAS 2000 image analyzer (Fuji Co., Tokyo, Japan). Glyceraldehyde-3-phosphate dehydrogenase (GAPDH) was used as an internal control.

Apoptosis and VEGF expression in the tumor. Apoptosis was detected with DNA fragmentation products that were stained by in situ 3'-end labeling [terminal deoxynucleotidyl transferase-mediated dUTP nick-labeling (TUNEL)] with paraffinembedded sections. In each tumor, the positive cells in 10 high-power fields at a magnification $\mathrm{x} 400$ were examined as described previously (23). For measurement of the VEGF protein level in the tumor, five tumors having the same size were chosen from each group, because a different size of tumor may cause different hypoxic conditions, which strongly induce VEGF (24). The tumor samples were prepared as described previously (25). After the protein concentration was equalized, the VEGF level was measured with an ELISA kit (R\&D Systems, Minneapolis, MN, USA) in accordance with the supplier's instructions.

In vitro proliferation and assay. The in vitro proliferation was determined by MTT assay as described elsewhere (25). The cell proliferation was quantified via conversion of tetrazolium, 3-(4,5-diethylthiazol-2-yl)-2,5-diphenyltetrazolium bromide (MTT) by cells cultured in 12-well plate. MTT was added to each well at a final concentration IFN- $\alpha$ and $-\beta\left(10,10^{2}\right.$, $10^{3} \mathrm{IU} / \mathrm{ml}$ ). After $4-\mathrm{h}$ incubation at $37^{\circ} \mathrm{C}$ with MTT, the untreated MTT and medium were removed, and $2 \mathrm{ml}$ of dimethyl sulfoxide were added to solubilize the MTT formazan. After gentle agitation for $10 \mathrm{~min}$, the optical density of each well, which is directly proportional to the number of living cells, was measured with a 540-nm filter. The absorbance was read with an ELISA plate reader $(n=6$ per group).

Statistical analysis. To assess the statistical significance of inter-group differences in the quantitative data, Bonferroni's multiple comparison test was performed after One-way ANOVA. This was followed by Barlett's test to determine the homology of variance. 


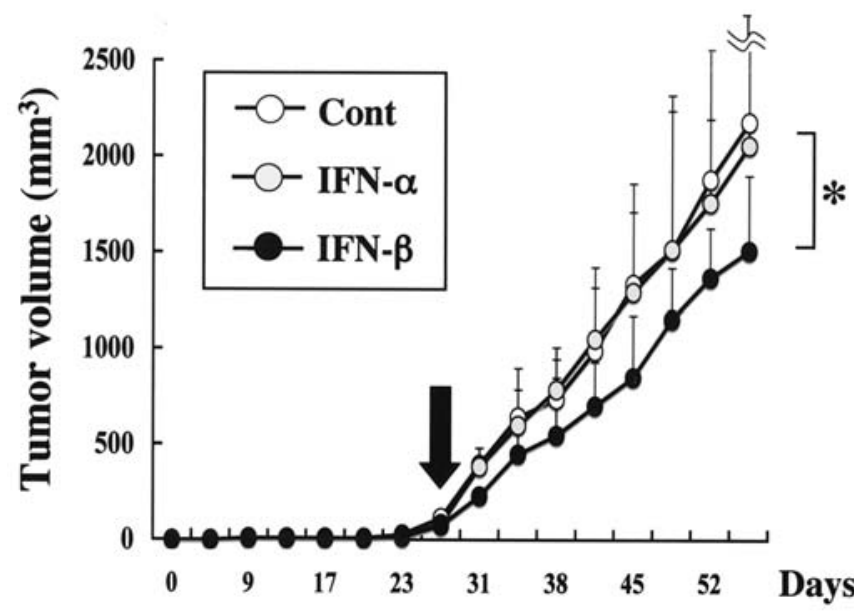

Figure 1. Effects of IFN- $\alpha$ and IFN- $\beta$ on the HCC growth. A clinically comparable low dose of IFN- $B$, even after the tumor was established, showed a marked inhibitory effect on HCC development as compared with the control group, whereas IFN- $\alpha$ treatment did not. The tumor volume was determined by calipers at the indicated time-points. Each point represents the mean \pm SD $(n=10)$. * Statistically significant difference between the indicated experimental groups $(\mathrm{p}<0.05)$. Cont, PBS-treated control group. INF- $\alpha$, IFN- $\beta$, IFN- $\alpha$ and IFN- $\beta$ treated groups at dose of $1 \times 10^{4} \mathrm{IU} /$ twice a week starting from day 14 (the black arrow), respectively.

\section{Results}

Effects of IFN- $\alpha$ and IFN- $\beta$ on HCC growth and neovascularization. We first examined the effects of clinically comparable doses of IFN- $\alpha$ and $-\beta$ on the HCC growth. As shown in Fig. 1, IFN- $\beta$ treatment, even after the tumor was established, showed a marked inhibitory effect on HCC development as compared with the control group $(\mathrm{p}<0.05)$, whereas IFN- $\alpha$ treatment did not. We next examined the effects of IFN $-\alpha$ and $-\beta$ on neovascularization in the tumor to elucidate whether or not the inhibitory effects of these agents are associated with alteration of angiogenesis. We performed a semi-quantitative RT-PCR analysis of the CD31 gene expression to evaluate neovascularization. As shown in Fig. 2, almost in parallel to the results of the HCC growth, the expression of CD31 in the IFN- $\beta$ treatment group was markedly suppressed as compared with the control group, whereas IFN- $\alpha$ treatment did not exert such an inhibitory effect. Both compounds at the current doses did not affect the healthy state parameters; e.g., the body weight during the experiment (data not shown).

Effects of IFN- $\alpha$ and IFN- $\beta$ on EC proliferation. To elucidate whether the differential tumoricidal effect between IFN- $\alpha$ and $-\beta$ via anti-angiogenesis was due to the cytotoxic sensitivity to the EC or not, we next examined the effects of IFN- $\alpha$ and $-\beta$ on EC proliferation in vitro. Both IFN- $\alpha$ and $-\beta$ inhibited the EC proliferation in a dose-dependent manner. However, the sensitivity was significantly different. IFN- $\beta$ had a marked inhibitory effect on EC proliferation even at a low dose $(10 \mathrm{IU} / \mathrm{ml})$, whereas IFN- $\alpha$ did not exhibit such an inhibitory effect even at a ten-times higher dose (100 IU/ml). IFN- $\alpha$ first exerted a significant inhibitory effect at $1,000 \mathrm{IU} / \mathrm{ml}$ although

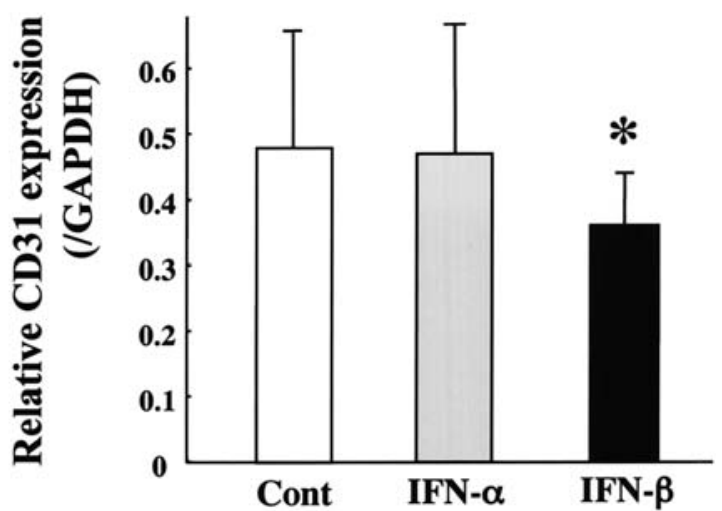

Figure 2. Effects of IFN- $\alpha$ and IFN- $\beta$ on the mRNA expressions of CD31. The mRNA expressions were examined by RT-PCR as described in Materials and methods. IFN- $\beta$ showed a significant inhibitory effect on neovascularization in the tumor as compared with the control group almost in parallel with the suppression of tumor growth, whereas IFN- $\alpha$ treatment did not. Each bar represents the mean \pm SD $(n=5)$. *Statistically significant difference between the indicated experimental groups $(\mathrm{p}<0.05)$. Cont, PBS-treated control group. INF- $\alpha$, IFN- $\beta$, IFN- $\alpha$ and IFN- $\beta$ treated groups, respectively.

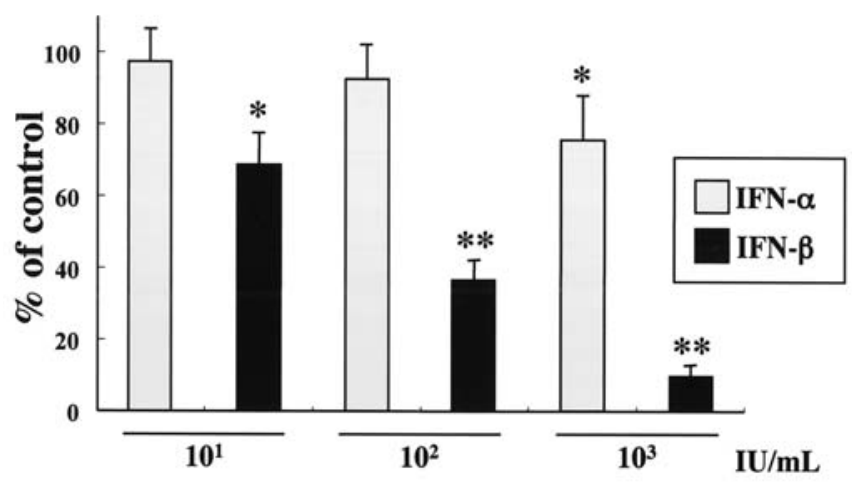

Figure 3. Effects of IFN- $\alpha$ and IFN- $\beta$ on the EC proliferation in vitro. Cell proliferation was measured by MTT assay as described in Materials and methods. Although both IFN- $\alpha$ and IFN- $\beta$ inhibited the EC proliferation in a dose-dependent manner, IFN- $B$ exerted a more potent inhibitory effect than IFN- $\alpha$. Each bar represents the mean \pm SD $(n=5) .{ }^{*}$ and ${ }^{* *}$ indicate statistically significant differences between the indicated experimental groups ( $\mathrm{p}<0.05$ and 0.01 , respectively). IFN- $\alpha$ and IFN- $\beta\left(10,10^{2}, 10^{3}\right)$ : IFN- $\alpha$ and IFN- $\beta$-treated groups at doses of $10,10^{2}, 10^{3} \mathrm{IU} / \mathrm{ml}$, respectively.

its magnitude was very low as compared with IFN-B (Fig. 3). Neither IFN- $\alpha$ nor IFN- $\beta$ suppressed the HCC proliferation in vitro even at a high dose $(1000 \mathrm{IU} / \mathrm{ml})$ (data not shown).

Effect of the host p53 status on the tumoricidal effect of IFN. We first confirmed the host p53 status of BNL and PLC by ELISA (Roche, Germany; and Oncogene, Cambridge, MA, USA), and found that BNL and PLC exerted p53-wild and mutant status, respectively (data not shown) as described previously (26). Since only IFN- $\beta$ exerted an inhibitory effect on the HCC growth at the current dose, we employed IFN- $B$ in the following study. We then examined whether the tumoricidal effect of IFN- $\beta$ was altered by the host p53 status or not. As shown in Fig. 4, IFN- $\beta$ exerted a marked inhibitory effect on 
A

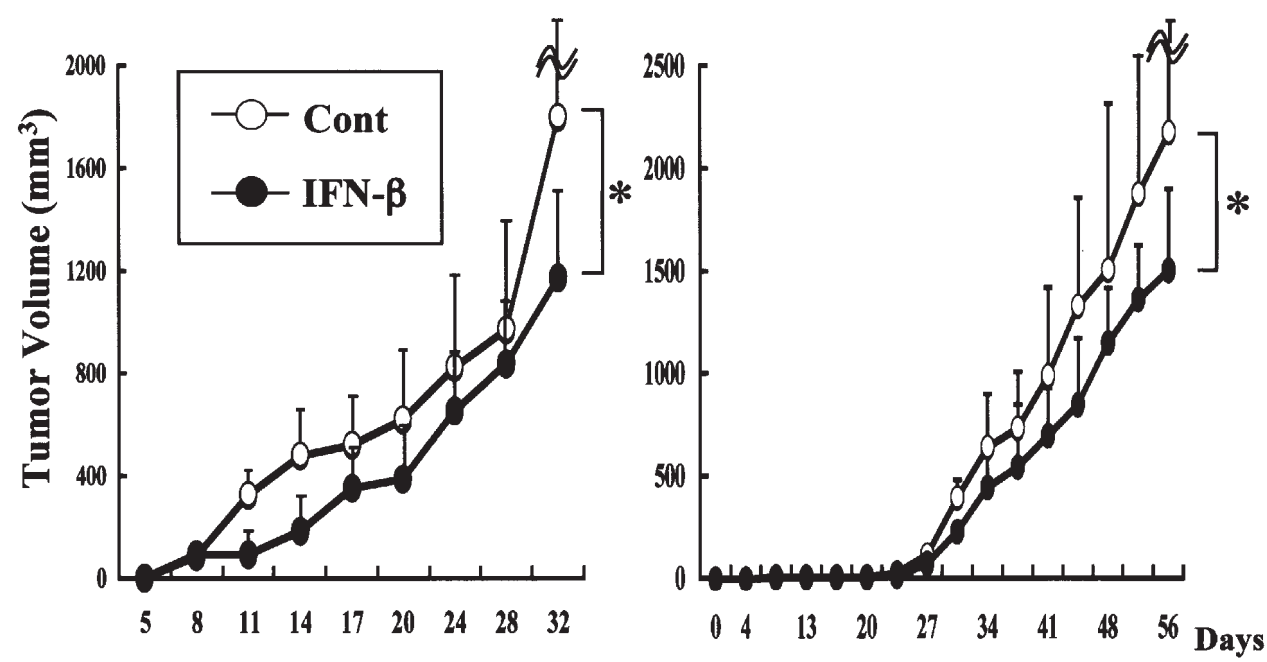

Figure 4. Correlation between the tumoricidal effect of IFN- $\beta$ and the host p53 status in the p53-wild (A) and -mutant HCC (B). Since only IFN- $\beta$ exerted an inhibitory effect on the HCC growth at the current dose, we employed IFN- $\beta$ in this study. IFN- $\beta$ revealed a marked inhibitory effect on the HCC growth regardless of the host p53 status; i.e., BNL-p53-wild and PLC-p53-mutant tumors. Each point represents the mean \pm SD (n=10). "Statistically significant difference between the indicated experimental groups $(\mathrm{p}<0.05)$. Cont, PBS-treated control group. IFN- $\beta$, IFN- $\beta$ treated group.

A

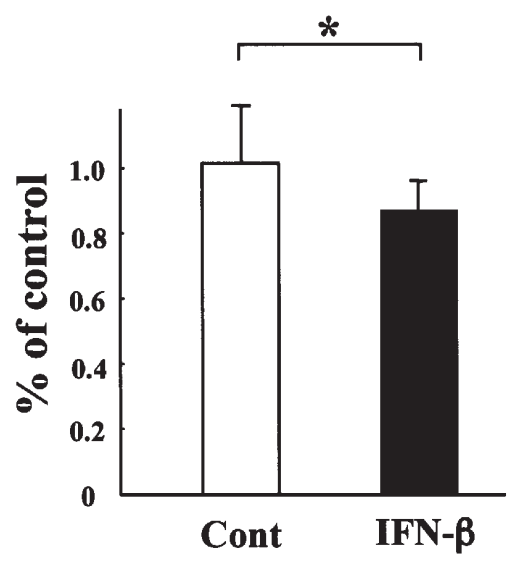

B

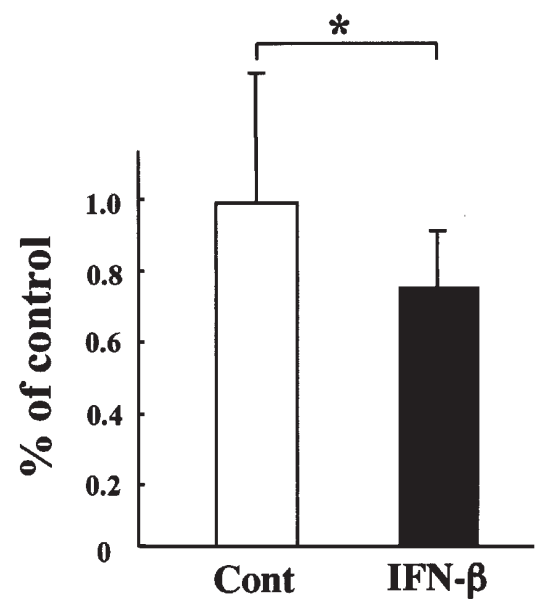

Figure 5. Effects of IFN- $\alpha$ and IFN- $\beta$ on the mRNA expressions of CD31. The mRNA expressions were examined by RT-PCR as described in Materials and methods. Similar to the tumoricidal effect, IFN-ß significantly suppressed the intra-tumoral neovascularization both in the BNL (A, p53-wild) and PLC (B, p53-mutant) tumors almost along with inhibition of the tumor growth. Each bar represents the mean \pm SD $(n=5)$. ${ }^{*}$ tatistically significant difference between the indicated experimental groups $(\mathrm{p}<0.05)$. Cont, PBS-treated control group. IFN- $\beta$, IFN- $\beta$ treated group.

the HCC growth regardless of the host p53 status; i.e., BNLp53-wild and PLC-p53-mutant tumors.

Angiogenesis, apoptosis, and VEGF in the tumor. To elucidate the possible mechanism of the inhibitory effect of IFN-B, we elucidated the neovascularization, apoptosis, and VEGF expression level in the BNL and PLC tumors. Similar to the tumoricidal effect, IFN- $\beta$ significantly suppressed the intratumoral neovascularization in both the BNL (p53-wild) and PLC (p53-mutant) tumors almost along with inhibition of the tumor growth (Fig. 5). On the other hand, the TUNEL-positive apoptotic cells markedly increased in both the BNL and PLC tumors by treatment with IFN- $\beta$. The incidence of apoptosis in the tumor almost corresponded to the effect of tumor development inhibition (Fig. 6). We also examined the VEGF protein level in the tumor, and found that the VEGF expression was significantly attenuated by IFN- $\beta$ in both the BNL and PLC tumor indicating that the anti-angiogenic activity by IFN was likely, at least partly, to mediate the VEGF inhibition (Fig. 7). 
A

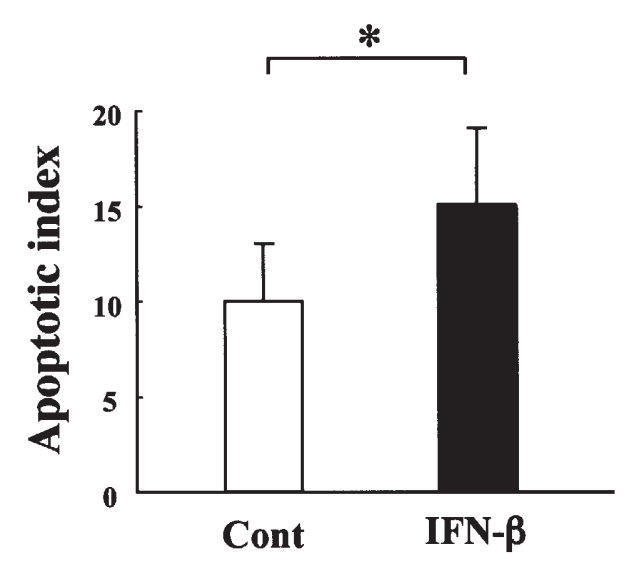

B

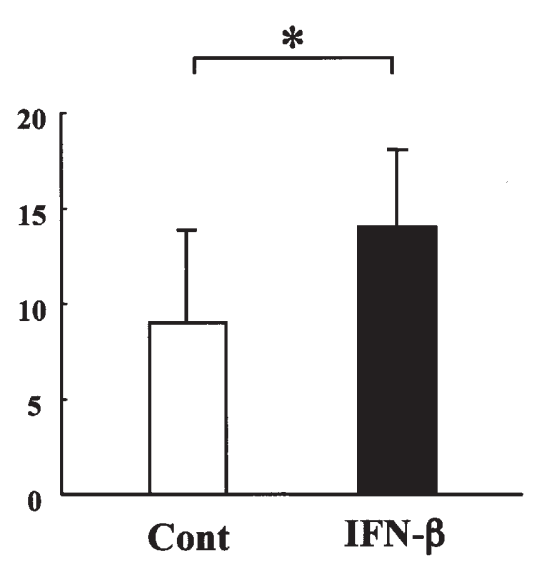

Figure 6. Effects of IFN- $\alpha$ and IFN- $B$ on the apoptotic index in HCC. The apoptotic index was elucidated by immunohistochemical analysis as described in Materials and methods. The TUNEL-positive apoptotic cells markedly increased both in the BNL (A, p53-wild) and PLC (B, p53-mutant) tumors by treatment with IFN-B. The incidence of apoptosis in the tumor almost corresponded to the effect of tumor development inhibition. Each bar represents the mean \pm SD $(n=5)$. *Statistically significant difference between the indicated experimental groups $(\mathrm{p}<0.05)$. Cont, PBS-treated control group. IFN- $\beta$, IFN- $\beta$ treated group.

A

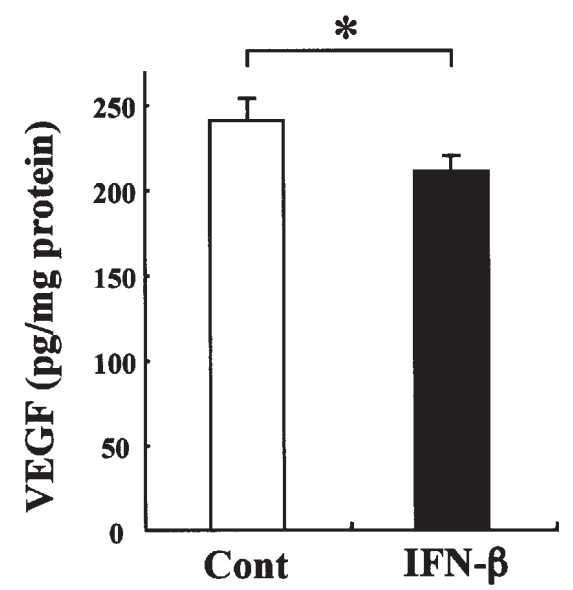

B

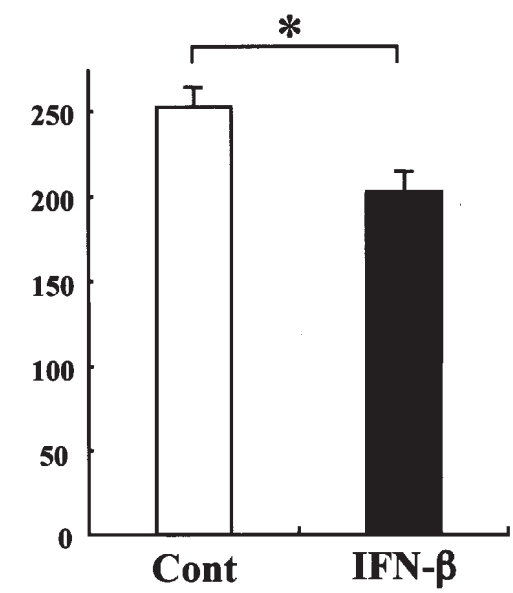

Figure 7. Effects of IFN- $\alpha$ and IFN- $\beta$ on the VEGF expression in HCC. The VEGF protein level was measured by ELISA as described in Materials and methods. IFN- 3 significantly suppressed the intra-tumoral VEGF expression both in the BNL (A, p53-wild) and PLC (B, p53-mutant) tumors almost along with inhibition of the tumor growth, indicating that the anti-angiogenic activity of IFN was likely, at least partly, to mediate the VEGF inhibition. Each bar represents the mean $\pm \mathrm{SD}(\mathrm{n}=5)$. ${ }^{*}$ Statistically significant difference between the indicated experimental groups (p<0.05). Cont, PBS-treated control group. IFN-B, IFN- 3 treated group.

\section{Discussion}

IFN was originally identified because of its function in antiviral host defense, and type-I IFN, IFN- $\alpha$, and IFN- $\beta$ are widely used for eradication of hepatitis viruses in patients with chronic hepatitis (8). Recent studies have revealed that IFN also exerted a tumoricidal effect in several types of tumors, including HCC $(10,27)$. Most of these studies showed that the tumoricidal effect of IFN was due to direct cytotoxicity against the tumor cells at relatively high doses (28). However, the long-term administration of high doses of IFN is unacceptable for the patients with chronic liver diseases. In the clinical practice, continuous administration of high doses of IFN resulted in several symptoms including fatigue, anorexia, weight loss, dizziness, and severe hematological disorders (28).

Since therapies aiming at destruction of the tumor vasculature can achieve rapid regression of the experimental tumors, the anti-angiogenic therapy has been under investigation around the world, including the use of gene therapy, antiangiogenic recombinant proteins, monoclonal antibodies, and various drugs $(29,30)$. The first anti-angiogenic agent approved in the clinical practice was Bevacizumab (Avastin), a humanized version of the VEGF monoclonal antibody, in 
2004 (31). Bevacizumab exerted a significant beneficial effect in patients with metastatic colon cancer in combination with 5-FU-based chemotherapy regimens (32). Sorafinib is another anti-angiogenic agent which blocks Raf kinase and VEGFR-2 tyrosine kinase inhibitor (33). Sorafinib has been recently approved by the Food and Drug Administration in the United States of America for metastatic renal cell carcinoma, and it is currently under clinical trials for other organ malignancies (34). It has been reported that the Raf activity and VEGFR-2 expression were significantly up-regulated in the human HCC tumor samples (35). In Japan, a clinical trial of Sorafinib against the HCC cumulative recurrence after TAE has recently been launched. However, it appears that some more time is required before Sorafinib can be applied widely in the clinical practice for HCC. An alternative approach may be to find a clinically available compound that also shows an anti-angiogenic activity until these new drugs become widely available. In the current study, we showed that clinically comparable low doses of IFN significantly suppressed the HCC growth via anti-angiogenesis. Although both IFN- $\alpha$ and IFN- $\beta$ utilized the same type receptor, IFN- $\beta$ exerted a more potent anti-angiogenic activity than IFN- $\alpha$. Several studies have shown that the signaling cascades of IFN- $\alpha$ and IFN- $\beta$ are different in several types of cells including the HCC cells $(36,37)$. IFN- $\alpha$ and IFN- $\beta$ activated ERK $1 / 2$ and or AKT independently of modulating the proliferation of HCC cells (38). Since the toxic sensitivities of IFN- $\alpha$ and IFN- $\beta$ for the EC were remarkably different, there may be a possibility that different signaling cascades were utilized for each IFN in the EC.

Some recent studies have revealed a close relationship between the p53 status of the host tumor cells and the sensitivity for anti-angiogenic therapy (20). It has been reported that mice bearing tumors derived from p53 (-/-) HCT116 human colorectal cancer cells were less responsive to antiangiogenic combination therapy than mice bearing isogenic p53 (+/+) tumors $(39,40)$. The less biological activities of IFN under the condition of p53 (-/-) for tumor suppression and antiviral defense have also been reported (20). In this study, we observed that the anti-angiogenic activity of IFN was not significantly different regardless of the host p53 status. At this time, the exact reason for this discrepancy is still obscure. However, it has been reported that inhibition of p53 during angiogenesis does not affect the extent of new vessel formation (41). IFN also prevented and delayed rat hepatocarcinogenesis through p53-independent pathway (42). Furthermore, it has been recently reported that the p53 status could not estimate the prognosis of the HCC patients, whereas angiogenic factors such as VEGF showed a positive correlation with the prognosis $(43,44)$.

Taken together, there may be a possibility that the antiangiogenic activity of IFN is tumor cell type specific. Alternatively, the sensitivity of the newly developed EC in HCC for the anti-angiogenic agents may be independent of the p53 status. Further studies are required to elucidate the exact mechanism in the future.

Augmentation of apoptosis without affecting the tumor cell proliferation is known as one of the characteristic features of the effectiveness with anti-angiogenic therapy (45). We observed that the significant increase in apoptosis in HCC by treatment with IFN almost corresponded to the effect of tumor growth inhibition, and that IFN did not alter the proliferation of HCC cells in vitro. These results suggested that IFN induced tumor inhibition by means of increasing apoptosis in the tumor but not via suppression of the tumor cell proliferation. It is an important issue to elucidate whether the low dose of IFN directly induced the tumor cell apoptosis or not. We observed that, even at a low dose, IFN significantly inhibited the EC proliferation in vitro, whereas it did not affect the HCC cell proliferation. Furthermore, IFN attenuated the expression of VEGF in the tumor, which is known as a survival factor for the EC (46). Taken together, IFN may first induce EC apoptosis, with subsequent secondary apoptosis in the tumor. Alternatively, IFN acts on both HCC cells and EC by down-regulation of the intra-tumoral VEGF and inhibition of EC proliferation, respectively. These biphasic effects of IFN may also play an important role in the tumoricidal effect against $\mathrm{HCC}$.

In conclusion, IFN exerted a marked inhibitory effect on the HCC growth, even after the tumor was established, via anti-angiogenesis along with suppression of VEGF and augmentation of apoptosis in the tumor. IFN- $\beta$ significantly revealed more potent tumoricidal effect than IFN- $\alpha$, and a clinically comparable low dose of IFN- 3 inhibited the HCC growth regardless of the host p53 status. A cautious approach may be also required in the clinical practice since even in the same IFN subclass (class-I), IFN- $\alpha$ and IFN- $\beta$ exert tumoricidal effects of different magnitudes on HCC.

\section{References}

1. Llovet JM, Burroughs A and Bruix J: Hepatocellular carcinoma. Lancet 362: 1907-1917, 2003.

2. Iwamiya T, Sawada S and Ohta Y: Repeated arterial infusion chemotherapy for inoperable hepatocellular carcinoma using an implantable drug delivery system. Cancer Chemother Pharmacol 33: S134-S138, 1994.

3. Llovet JM: Updated treatment approach to hepatocellular carcinoma. J Gastroenterol 40: 225-235, 2005.

4. Sakon M, Nagano H, Dono K, et al: Combined intraarterial 5fluorouracil and subcutaneous interferon-alpha therapy for advanced hepatocellular carcinoma with tumor thrombi in the major portal branches. Cancer 94: 435-442, 2002.

5. Obi S, Yoshida H, Toune R, et al: Combination therapy of intraarterial 5-fluorouracil and systemic interferon-alpha for advanced hepatocellular carcinoma with portal venous invasion. Cancer 106: 1990-1997, 2006.

6. Hertzog PJ, Hwang SY and Kola I: Role of interferons in the regulation of cell proliferation, differentiation, and development. Mol Reprod Dev 39: 226-232, 1994.

7. Poynard T, Yuen MF, Ratziu V and Lai CL: Viral hepatitis C. Lancet 362: 2095-2100, 2003.

8. Hayashi $\mathrm{N}$ and Takehara $\mathrm{T}$ : Antiviral therapy for chronic hepatitis C: past, present, and future. J Gastroenterol 41: 17-27, 2006.

9. Nishiguchi S, Shiomi S, Nakatani S, et al: Prevention of hepatocellular carcinoma in patients with chronic active hepatitis $\mathrm{C}$ and cirrhosis. Lancet 357: 196-197, 2001.

10. Shiratori Y, Ito Y, Yokosuka O, et al: Antiviral therapy for cirrhotic hepatitis $\mathrm{C}$ : association with reduced hepatocellular carcinoma development and improved survival. Ann Intern Med 142: 105-114, 2005.

11. Kerbel RS: Tumor angiogenesis: past, present and the near future. Carcinogenesis 21: 505-515, 2000.

12. Guo RP, Zhong C, Shi M, et al: Clinical value of apoptosis and angiogenesis factors in estimating the prognosis of hepatocellular carcinoma. J Cancer Res Clinical Oncol 132: 547-555, 2006.

13. Iavarone M, Lampertico P, Iannuzzi F, et al: Increased expression of vascular endothelial growth factor in small hepatocellular carcinoma. J Viral Hepatitis 14: 133-139, 2007. 
14. Yoshiji H, Kuriyama S, Yoshii J, et al: Vascular endothelial growth factor tightly regulates in vivo development of murine hepatocellular carcinoma cells. Hepatology 28: 1489-1496, 1998.

15. Albini A, Marchisone C, Del Grosso F, et al: Inhibition of angiogenesis and vascular tumor growth by interferon-producing cells: a gene therapy approach. Am J Pathol 156: 1381-1393, 2000.

16. Rozera C, Carlei D, Lollini PL, et al: Interferon (IFN)-beta gene transfer into TS/A adenocarcinoma cells and comparison with IFN-alpha: differential effects on tumorigenicity and host response. Am J Pathol 154: 1211-1222, 1999.

17. Da Silva AJ, Brickelmaier M, Majeau GR, et al: Comparison of gene expression patterns induced by treatment of human umbilical vein endothelial cells with IFN-alpha $2 b$ vs. IFN-beta $1 \mathrm{a}$ : understanding the functional relationship between distinct type I interferons that act through a common receptor. J Interferon Cytokine Res 22: 173-188, 2002.

18. Hayashi H, Sugio K, Matsumata T, Adachi E, Takenaka K and Sugimachi K: The clinical significance of p53 gene mutation in hepatocellular carcinomas from Japan. Hepatology 22: 1702-1707, 1995.

19. Teramoto T, Satonaka K, Kitazawa S, Fujimori T, Hayashi K and Maeda S: p53 gene abnormalities are closely related to hepatoviral infections and occur at a late stage of hepatocarcinogenesis. Cancer Res 54: 231-235, 1994.

20. Takaoka A, Hayakawa S, Yanai $\mathrm{H}$, et al: Integration of interferon-alpha/beta signalling to p53 responses in tumour suppression and antiviral defence. Nature 424: 516-523, 2003.

21. Yoshiji H, Kuriyama S, Ways DK, et al: Protein kinase C lies on the signaling pathway for vascular endothelial growth factormediated tumor development and angiogenesis. Cancer Res 59: 4413-4418, 1999

22. Noguchi R, Yoshiji H, Kuriyama S, et al: Combination of interferon-beta and the angiotensin-converting enzyme inhibitor, perindopril, attenuates murine hepatocellular carcinoma development and angiogenesis. Clin Cancer Res 9: 6038-6045, 2003.

23. Yoshii J, Yoshiji H, Kuriyama S, et al: The copper-chelating agent, trientine, suppresses tumor development and angiogenesis in the murine hepatocellular carcinoma cells. Int J Cancer 94: 768-773, 2001 .

24. Shibuya M: Structure and function of VEGF/VEGF-receptor system involved in angiogenesis. Cell Struct Funct 26: 25-35, 2001.

25. Yoshiji H, Kuriyama S, Yoshii J, et al: Synergistic effect of basic fibroblast growth factor and vascular endothelial growth factor in murine hepatocellular carcinoma. Hepatology 35: 834-842, 2002.

26. Chiang LC, Ng LT, Lin IC, Kuo PL and Lin CC: Anti-proliferative effect of apigenin and its apoptotic induction in human Hep G2 cells. Cancer Lett 237: 207-214, 2006.

27. Yoshida H, Tateishi R, Arakawa Y, et al: Benefit of interferon therapy in hepatocellular carcinoma prevention for individual patients with chronic hepatitis C. Gut 53: 425-430, 2004.

28. Wang L, Tang ZY, Qin LX, et al: High-dose and long-term therapy with interferon-alfa inhibits tumor growth and recurrence in nude mice bearing human hepatocellular carcinoma xenografts with high metastatic potential. Hepatology 32: 43-48, 2000.

29. Kerbel RS: Clinical trials of antiangiogenic drugs: opportunities, problems, and assessment of initial results. J Clin Oncol 19: S45-S51, 2001.
30. Carmeliet P: Angiogenesis in life, disease and medicine. Nature 438: 932-936, 2005 .

31. Ferrara N: VEGF as a therapeutic target in cancer. Oncology 69 (Suppl. 3): 11-16, 2005.

32. Kabbinavar FF, Hambleton J, Mass RD, Hurwitz HI, Bergsland E and Sarkar S: Combined analysis of efficacy: the addition of bevacizumab to fluorouracil/leucovorin improves survival for patients with metastatic colorectal cancer. J Clin Oncol 23: 3706-3712, 2005.

33. Wilhelm SM, Carter C, Tang L, et al: BAY 43-9006 exhibits broad spectrum oral antitumor activity and targets the RAF/ MEK/ERK pathway and receptor tyrosine kinases involved in tumor progression and angiogenesis. Cancer Res 64: 7099-7109, 2004.

34. Gollob JA, Wilhelm S, Carter C and Kelley SL: Role of Raf kinase in cancer: therapeutic potential of targeting the Raf/ MEK/ERK signal transduction pathway. Semin Oncol 33: 392-406, 2006.

35. Abou-Alfa GK, Schwartz L, Ricci S, et al: Phase II study of sorafenib in patients with advanced hepatocellular carcinoma. $\mathbf{J}$ Clin Oncol 24: 4293-4300, 2006.

36. Murata M, Nabeshima S, Kikuchi K, Yamaji K, Furusyo N and Hayashi J: A comparison of the antitumor effects of interferonalpha and beta on human hepatocellular carcinoma cell lines. Cytokine 33: 121-128, 2006.

37. Damdinsuren B, Nagano H, Wada H, et al: Stronger growthinhibitory effect of interferon (IFN)-beta compared to IFN-alpha is mediated by IFN signaling pathway in hepatocellular carcinoma cells. Int J Oncol 30: 201-208, 2007.

38. Matsumoto K, Okano J and Murawaki Y: Differential effects of interferon alpha- $2 \mathrm{~b}$ and beta on the signaling pathways in human liver cancer cells. J Gastroenterol 40: 722-732, 2005.

39. Bunz F, Dutriaux A, Lengauer C, et al: Requirement for p53 and p21 to sustain G2 arrest after DNA damage. Science 282: 1497-1501, 1998.

40. Yu JL, Rak JW, Coomber BL, Hicklin DJ and Kerbel RS: Effect of p53 status on tumor response to antiangiogenic therapy. Science 295: 1526-1528, 2002.

41. Bordel R, Laschke MW, Menger MD and Vollmar B: Inhibition of p53 during physiological angiogenesis in the hamster ovary does not affect extent of new vessel formation but delays vessel maturation. Cell Tissue Res 320: 427-435, 2005.

42. Nakaji M, Yano Y, Ninomiya T, et al: IFN-alpha prevents the growth of pre-neoplastic lesions and inhibits the development of hepatocellular carcinoma in the rat. Carcinogenesis 25: 389-397, 2004.

43. Cascinu S, Graziano F, Catalano V, et al: Vascular endothelial growth factor and p53 expressions in liver and abdominal metastases from colon cancer. Tumour Biol 24: 77-81, 2003.

44. Fondevila C, Metges JP, Fuster J, et al: p53 and VEGF expression are independent predictors of tumour recurrence and survival following curative resection of gastric cancer. Br J Cancer 90: 206-215, 2004.

45. Ferrara $\mathrm{N}$ and Kerbel RS: Angiogenesis as a therapeutic target. Nature 438: 967-974, 2005

46. Wu WZ, Sun HC, Shen YF, et al: Interferon alpha 2a downregulates VEGF expression through PI3 kinase and MAP kinase signaling pathways. J Cancer Rese Clinical Oncol 131: 169-178, 2005 . 